\title{
Feasibility of Intravenous Flat Panel Detector CT Angiography for Intracranial Arterial Stenosis
}

\author{
J.S. Jeon, S.H. Sheen, G.J. Hwang, H.C. Kim, and B.J. Kwon
}

\begin{abstract}
BACKGROUND AND PURPOSE: IV FDCT angiography is an emerging technology for the detection of intracranial vascular disease. This study was conducted to determine the feasibility of IV FDCT in estimating major atherosclerotic intracranial arterial stenosis with DSA as the reference.
\end{abstract}

MATERIALS AND METHODS: DSA and IV FDCT were performed simultaneously in patients with transient ischemic attack or acute cerebral infarction. The degree and length of stenosis were measured. The stenotic vessels were categorized into 4 groups by the grade of stenosis: normal $(<30 \%)$, mild $(30 \%-49 \%)$, moderate $(50 \%-69 \%)$, or severe $(>70 \%)$. The vessels of the normal group were excluded from analysis to reduce spectrum bias. Measurement of vessels was recorded by using an electric ruler by a qualified endovascular neurosurgeon and a neuroradiologist.

RESULTS: Eight hundred forty-two vessel segments in 69 patients were calculated. Mild ( $n=56)$, moderate $(n=47)$ and severe stenosis ( $n=46$ ) groups were analyzed. IV FDCT had a sensitivity of $97.6 \%$, specificity of $96.9 \%$, and negative predictive value of $96.9 \%$ for detecting $\geq 50 \%$ stenosis and respective values of $91.9 \%, 98.2 \%$, and $97.4 \%$ for depicting $\geq 70 \%$ stenosis. The difference of stenotic length between the 2 tests was not significant as an increase in the severity of stenosis (Spearman rank correlation test; $r=-0.12, P=.13$ ).

CONCLUSIONS: IV FDCT can be a feasible alternative as a noninvasive method for evaluating stenosis of the major intracranial arteries.

ABBREVIATIONS: $\mathrm{Al}$ = segment of the anterior cerebral artery; $\mathrm{Cl}=$ confidence interval; $\mathrm{FDCT}$ = flat panel detector $\mathrm{CT} ; \mathrm{Ml}$ or $\mathrm{M2}=$ segments of the middle cerebral artery; $\mathrm{Pl}=$ segment of the posterior cerebral artery; $\mathrm{V} 4=$ segment of the vertebral artery

$\mathbf{T}$ he stenosis of atherosclerotic major intracranial arteries is an important cause of ischemic stroke. ${ }^{1}$ Although cerebral angiography has been regarded as the criterion standard for depicting vascular stenosis, noninvasive tests, including MRA, CTA, and transcranial Doppler sonography, have been conducted first, due to a potential risk of stroke and procedural complications. ${ }^{2,3}$

Recently, IV FDCT has been shown to have a high resolution that is similar to that of multidetector row CT angiography ${ }^{4}$ and has been suggested as an alternative tool for evaluating acute cerebral ischemia. ${ }^{5}$ Many trials have been conducted to investigate the accuracy of each noninvasive test compared with cerebral an-

Received December 28, 2011; accepted after revision April 16, 2012.

From the Departments of Neurosurgery (J.S.J., S.H.S., G.J.H.) and Neuroradiology (H.C.K.), Hallym University College of Medicine, Chun Cheon Sacred Heart Hospital, Chun Cheon, Korea; and Department of Neuroradiology (B.J.K.), Kwandong University College of Medicine, Myongji Hospital, Goyang, Korea.

Please address correspondence to Seung Hun Sheen, MD, Department of Neurosurgery, Hallym University College of Medicine, Chun Cheon Sacred Heart Hospital, Kyo-Dong, Chun Cheon, 200-704, Korea; e-mail: nssheen@gmail.com

http://dx.doi.org/10.3174/ajnr.A3301 giography, but to the best of our knowledge, no study has directly compared the degree and length of intracranial arterial stenosis between DSA and IV FDCT.

This study was undertaken to evaluate the feasibility of IV FDCT in estimating atherosclerotic stenosis severity and the length of the major intracranial arteries with DSA as the reference standard.

\section{MATERIALS AND METHODS}

This study was approved by the local ethics committee. This prospective analysis was performed in patients who concurrently had undergone DSA and IV FDCT from January 2009 to December 2011 at a single center. Patients with TIA or acute cerebral ischemia were included. Eight hundred forty-two vessel segments in 69 patients were measured.

After checking routine conventional angiography, 3D rotational angiography was performed in cases of vascular stenosis, and additional studies were performed with the guidance of a working projection of 3D angiography. IV FDCT was conducted simultaneously. Maximum-intensity-projection images obtained by IV FDCT were rotated and reconstructed for the same working 
views. In difficult cases that were not reconstructed from IV FDCT data, anterior and lateral projection images of DSA were matched with coronal and sagittal directional images of IV FDCT, respectively, for analysis.

Every vessel diameter, the degree of stenosis, and stenosis length were recorded by using an electric ruler by a qualified endovascular neurosurgeon and a neuroradiologist blinded to clinical information. The vessel diameter of the bilateral supraclinoid internal carotid artery, A1, M1, M2, P1, and V4, which was located just proximal to the posterior inferior cerebellar artery and the midpoint of the basilar artery, was measured. Percentage stenosis was determined as $[(1-(D$-stenosis/D-normal $)] \times 100$, where $\mathrm{D}$-stenosis was the most severe stenosis and D-normal was the diameter of proximal normal artery. The formula was used for measuring the degree of stenosis. ${ }^{6}$ The locations of stenotic segments were divided by direction (horizontal versus vertical) and circulation (anterior versus posterior). Horizontal directional components comprised A1, M1, and P1, and anterior circulation comprised ICA, A1, M1, and M2. Lesion length was defined as the distance from proximal to distal of stenotic vessel.

A disagreement of $>10 \%$ in stenosis prompted recalculation by a third reader. ${ }^{7}$ Cases of hypoplastic segments and cases in which visualization could be performed only through cross-fillings or that were not evident on DSA were excluded. ${ }^{7}$

Stenotic vessels were categorized into 4 groups by the degree of stenosis: normal $(<30 \%)$, mild $(30 \%-49 \%)$, moderate $(50 \%-$ $69 \%)$, or severe $(>70 \%)$. In particular, stenotic lesions graded as normal were excluded from analysis to avoid spectrum bias. ${ }^{8,9}$

\section{Image Acquisition}

Eighty milliliters of Pamiray 250 contrast agent (iopamidol; Dongkook Pharmaceutical, Seoul, Korea) mixed with $40 \mathrm{~mL}$ of saline was injected through an anterior cubital vein with a flow rate of $5 \mathrm{~mL} / \mathrm{s}$. IV FDCT parameters were 0.4 increment, $220^{\circ}$ total angle, 538 total projections, and radiation dose of $35 \mathrm{mGy}$ (manufacturer's information) with an acquisition time of a 20 -second rotation and an 18-second x-ray time delay. Postprocessing of the IV FDCT data was conducted on a Leonardo DynaCT dedicated workstation equipped with InSpace 3D software (Siemens, Erlangen, Germany). The images given as maximum-intensity-projection images in the axial, coronal, and sagittal planes with various section thicknesses, mainly $15 \mathrm{~mm}$, were reconstructed for analysis. An electronic ruler was used for estimating stenosis rate and length. Every IV FDCT and DSA were conducted with the Axiom Artis zee Biplane system (Siemens).

\section{Statistical Analysis}

Intraclass correlation, sensitivity, specificity, positive and negative predictive values, 95\% CIs, Bland-Altman analysis test, and a Spearman rank correlation test were used to assess the reliability of IV FDCT compared with DSA. Statistics were performed with the Statistical Package for the Social Sciences, Version 12 (SPSS, Chicago, Illinois) and MedCalc (www.medcalc.org).

\section{RESULTS}

Sixty-nine patients with 842 vessel segments were included. Their mean age was 59.1 years (range, $36-85$ years), and 31 patients
Table 1: Distribution of the stenotic vessel segments in each segment

\begin{tabular}{lrrrrrrrr}
\hline \multicolumn{1}{c}{ Stenosis } & ICA & A1 & M1 & M2 & P1 & BA & V4 & Total \\
\hline $30 \%-49 \%$ & 4 & 11 & 19 & 6 & 2 & 7 & 7 & 56 \\
$50 \%-69 \%$ & 5 & 7 & 17 & 2 & 2 & 9 & 5 & 47 \\
$70 \%-99 \%$ & 4 & 7 & 13 & 2 & 0 & 6 & 5 & 37 \\
Complete occlusion & 2 & 0 & 6 & 1 & 0 & 0 & 0 & 9 \\
Total & 15 & 25 & 55 & 11 & 4 & 22 & 17 & 149 \\
\hline
\end{tabular}

Note:-BA indicates basilar artery.

Table 2: Accuracy of IV FDCT in detecting severe stenosis (>70\%) compared with DSA ${ }^{\mathrm{a}}$

\begin{tabular}{lccc}
\hline & Seen on DSA & Not Seen on DSA & Total \\
\hline Seen on IV FDCT & 34 & 2 & 36 \\
Not seen on IV FDCT & 3 & 110 & 113 \\
Total & 37 & 112 & 149 \\
\hline
\end{tabular}

a The numbers given are vessel segments.

were male (44.9\%). The distribution of stenotic vessel segments is shown in Table 1.

The intraclass correlation for measuring $\geq 30 \%$ stenosis was 0.98 (95\% CI, 0.97-0.99). IV FDCT had a sensitivity of $97.6 \%$ (95\% CI, $91.7 \%-99.7 \%$ ), a specificity of $96.9 \%$ (95\% CI, $89.3 \%-$ $99.6 \%$ ), and a negative predictive value of $96.9 \%$ (95\% CI, 89.3\%$99.6 \%$ ) for detecting $\geq 50 \%$ stenosis with DSA as the reference. For identification of $\geq 70 \%$ stenosis, IV FDCT had a sensitivity of 91.9\% (95\% CI, 78.1\%-98.3\%), specificity of $98.2 \%$ (95\% CI, $93.7 \%-99.8 \%$ ), and negative predictive value of $97.4 \%$ (95\% CI, 92.4\%-99.5\%) (Table 2).

Bland-Altman plots showed good agreement between IV FDCT and DSA in both directions (horizontal versus vertical; Fig $1 A,-B$ ) and circulations (anterior versus posterior; Fig $1 C,-D$ ) in assessing the degree of stenosis. No tendency of over- or underestimation was found in the different stenotic groups.

Mean stenosis length was $6.92 \pm 2.2 \mathrm{~mm}$ in IV FDCT and $6.95 \pm 2.2$ in DSA. The difference of stenotic length between the 2 tests (IV FDCT and DSA, in millimeters) was not significantly different as an increase in the stenosis severity (Spearman rank correlation test, $r=-0.12 ; P=.13 ; 95 \% \mathrm{CI},-0.28-0.04$ ) (Fig $1 E)$.

\section{DISCUSSION}

Our study showed that IV FDCT has high sensitivity and specificity for detecting intracranial arterial stenosis and can be a reliable method for estimating stenosis length with the DSA standard. Many studies have been conducted to confirm the accuracy of stenosis by using 1 or combined noninvasive tests as the cerebral angiography reference. Good correlation between MRA and DSA for measuring major intracranial stenosis has been reported. ${ }^{10,11}$ However, MRA has some drawbacks, including the tendency to overestimate high-grade stenosis, being a more time-consuming procedure, and providing an underestimation or nonvisualization of stenosis located in the petrous or cavernous ICA. ${ }^{10}$

Several reports demonstrated high accuracy for detection of intracranial stenosis with CTA. ${ }^{7,8}$ CTA has been performed widely, reflecting its minimally invasive nature, fewer motion artifacts, and shorter time. However, the limitations of CTA include 

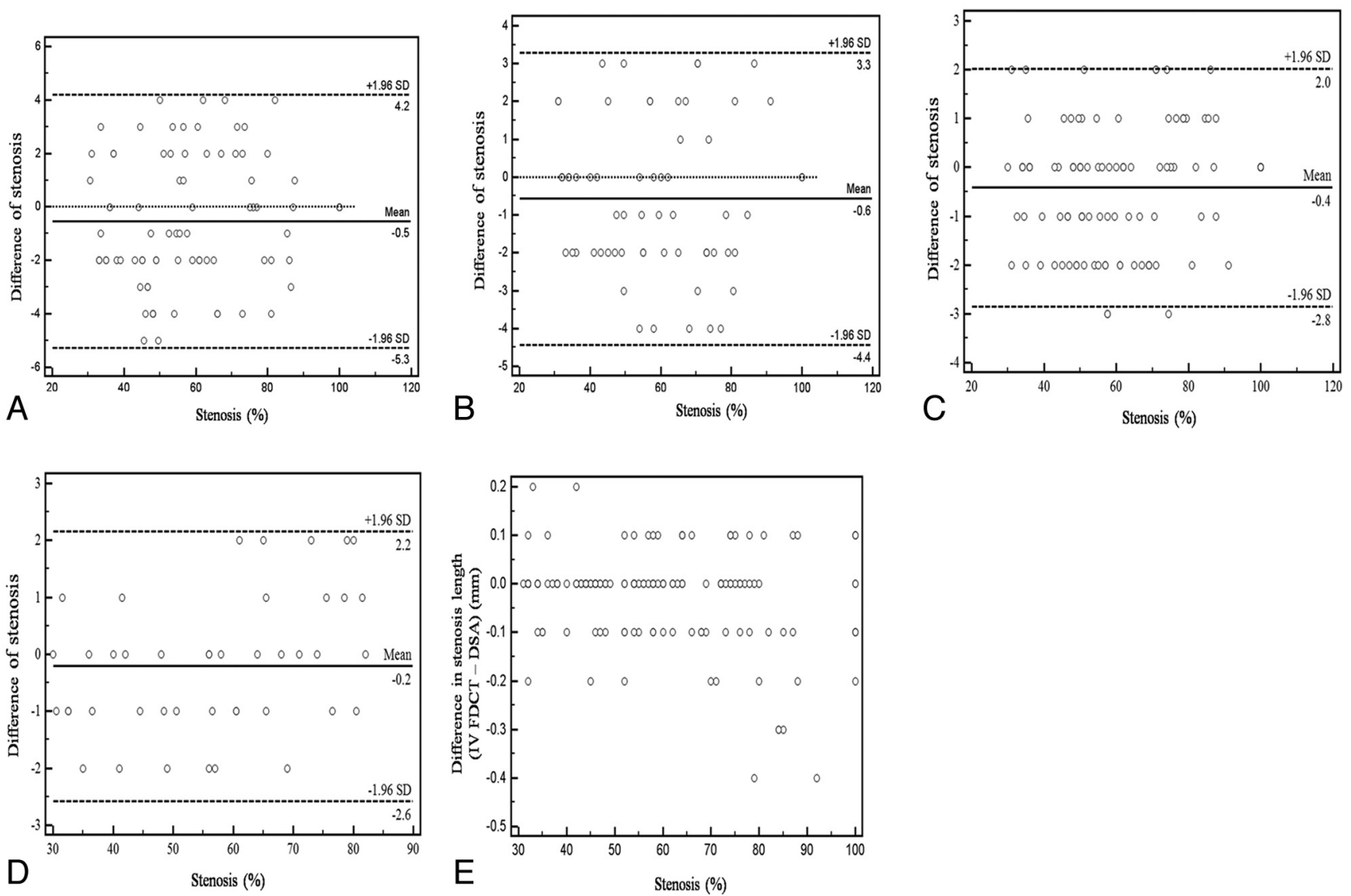

FIG 1. Bland-Altman plot shows excellent depiction of intracranial stenosis of the horizontal segments $(A)$, vertical segments $(B)$, anterior circulation (C), and posterior circulation ( $D$ ) by IV FDCT with DSA as the reference. Length discrepancy (IV FDCT-DSA, in millimeters) was found more often in higher stenosis but was not statistically significant ( $E$, Spearman rank correlation test; $r=-0.12, P=.13$ ).

the dose of radiation, poor visualization of collateral vessels, superimposition of bone and venous structures, and inaccurate stenosis measurement in the supraclinoid ICA. ${ }^{7,12}$

Hirai et $\mathrm{al}^{12}$ reported that the combination of MRA with CTA can improve sensitivity and specificity to $100 \%$ and $99 \%$, respectively, for detecting stenosis of $\geq 50$, but it is not a fully available procedure, considering its high cost and procedure time.

The SONIA trial ${ }^{13}$ revealed that high negative predictive values of the tests to exclude high-grade intracranial stenosis $(>70 \%)$ can be obtained with the combination of transcranial Doppler with MRA. Although the SONIA trial was a multicenter study and provided test cut-points when evaluating stenosis, the accuracy of transcranial Doppler sonography has been dependent on the operator's expertise ${ }^{14}$ and the results of transcranial Doppler sonography can vary among institutions.

IV FDCT is a novel technology for generating CT-like images from a biplane angiographic system. The distinctive feature of IV FDCT is its use in the neurointerventional setting without the need for patient transfer. Because the treatment of acute cerebral ischemia has a therapeutic time window, within 4.5 hours for intravenous thrombolysis ${ }^{15}$ or 6 hours for intra-arterial thrombolysis, ${ }^{16}$ it is crucial to shorten the time from the onset of symptoms to diagnosis and treatment for good clinical results. ${ }^{17}$ Therefore, in particular, patients who present with acute ischemic stroke may benefit from early detection with IV FDCT and instant endovascular treatment. ${ }^{5}$ In addition, IV FDCT allows the prompt detection of periprocedural complications. Conse- quently, operators can perform the endovascular intervention with more certainty and safety.

Images from IV FDCT can help set the revascularization treatment plan by providing valuable information on the accurate stenotic area, stenotic grade, and collateral circulation in the same acquisition, contrary to DSA. ${ }^{18}$ Blanc et al ${ }^{19}$ demonstrated the reliability of IV FDCT for assessing occlusion level, clot length, collateral degree, and patency of anterior and posterior communicating arteries.

Presently, no statistically significant difference in estimation of stenosis length was observed in higher stenosis grades (Spearman rank correlation test; $r=-0.12, P=.13$ ), but length discrepancy was more pronounced in higher stenosis. A possible explanation for that phenomenon may be the different hemodynamic effect on poststenotic segments between the 2 tests or spatial inhomogeneities such as surrounding tissues. ${ }^{20}$ Stenosis depiction with DSA can be influenced by dilution of the contrast medium during blood flow; and factors that may affect the flow velocity, including stenosis grade or injection technique, may lead to a difference in the measurement of the length of stenosis. The attenuation between diluted contrast agent administrated in IV FDCT and blood was more similar, and the diluted contrast agent may be able to fill the vessel more uniformly. ${ }^{21}$

Kamran et $\mathrm{al}^{20}$ suggested that IV FDCT can offer a better estimation of vascular structures as an even distribution and high resolution of contrast medium in comparison with 3D DSA. Thus, IV FDCT may produce less discrepancy in depicting vascu- 

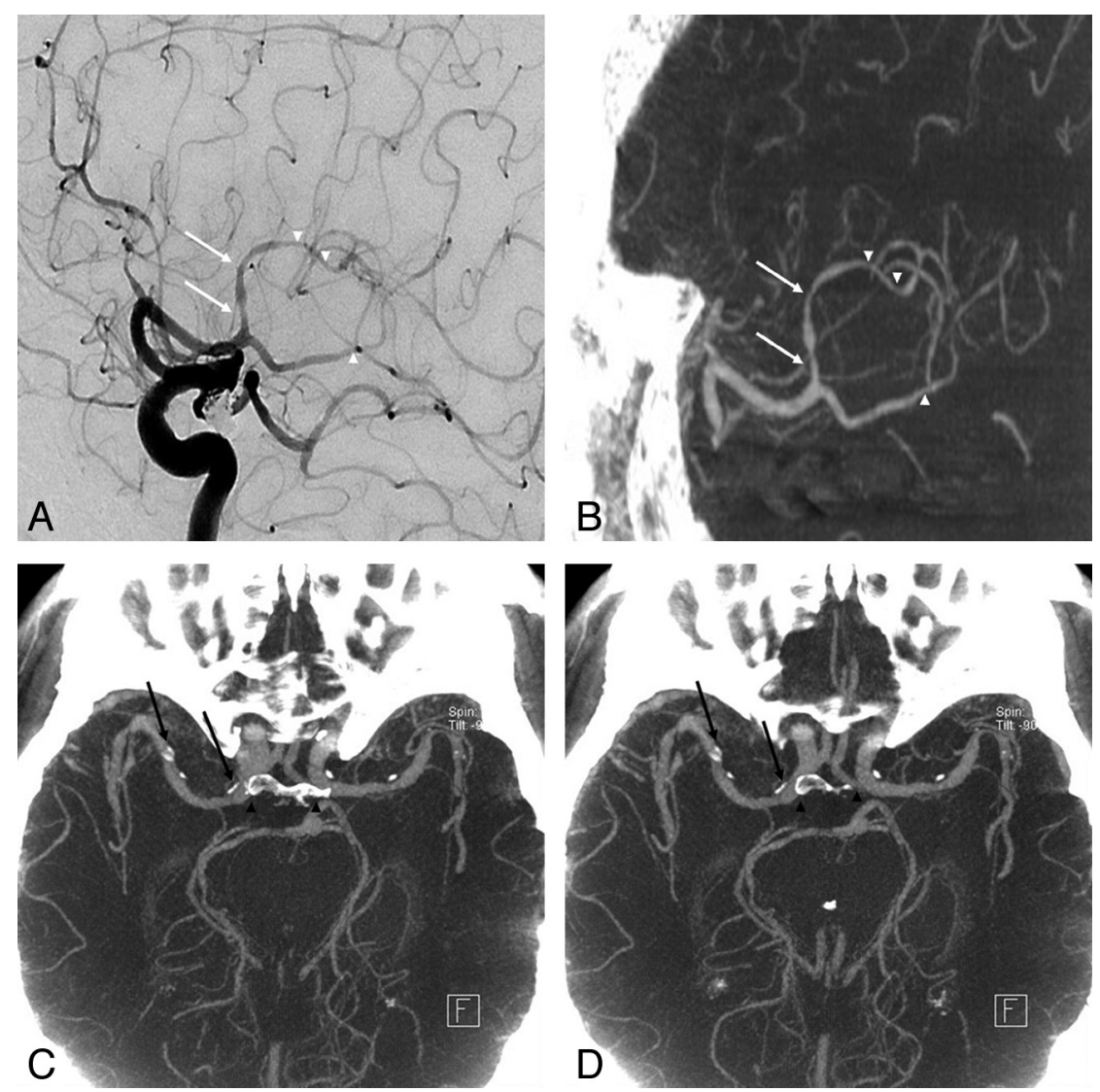

FIG 2. An 81-year-old woman who underwent coil embolization of an unruptured posterior communicating artery aneurysm 1 month before was referred due to a history of progressive left hemiparesis. $A$ and B, Good concordance was obtained for depicting stenosis at the proximal (arrows) and distal (arrowheads) M2 segment between DSA and IV FDCT. C and D, Axial maximum-intensity-projection image of IV FDCT allows a clear delineation of the vascular lumen from circumferential calcification (arrows) without superimposition of bone (arrowheads).
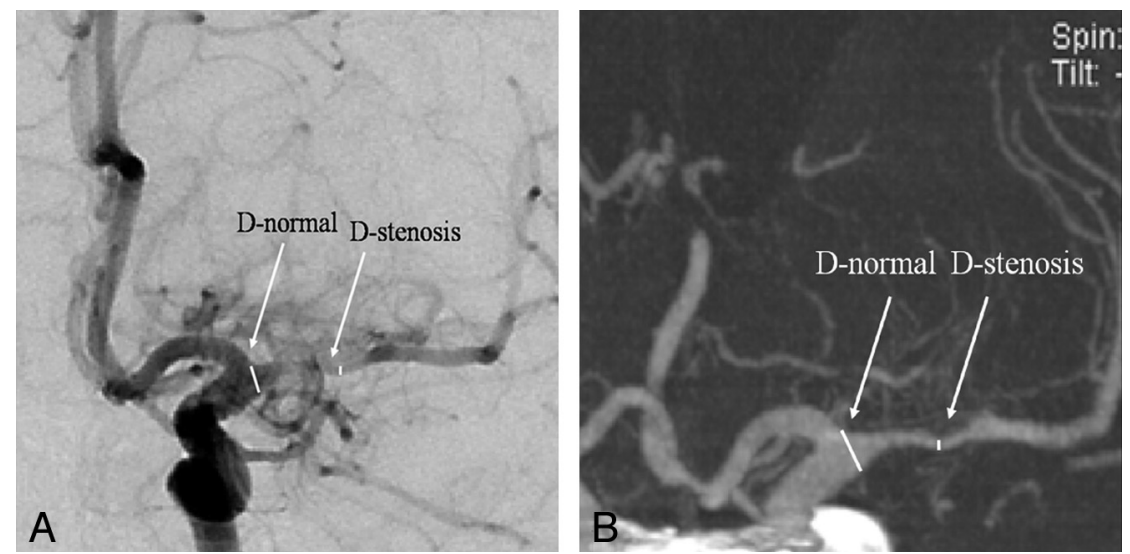

FIG 3. A 70-year-old woman was transferred for right-sided hemiparesis. A, Anteroposterior DSA of the left internal carotid artery shows severe stenosis $(76.2 \%)$ at the left Ml segment ( $D$-stenosis) with the beginning point of $M 1$ ( $D$-normal) as a reference point. $B$, Selective maximumintensity-projection image of IV FDCT reveals excellent concordance of severe stenosis (76.9\%) at the left M1 segment (arrows) with DSA as a reference.

lar morphology. In the case of assessing the stent lumen, IV FDCT allows more accurate postinterventional images than CTA. ${ }^{22,23}$

Although there is no statistic regarding the circumferential calcification in major intracranial arteries and distal arterial stenosis through a comparative study with CTA in this investigation, IV FDCT may clearly depict the vascular lumen with circumfer- ential calcification and distal stenosis with lesser superimposition of bone and venous structures (Fig 2).

Another advantage of IV FDCT is reduced radiation exposure. Our protocol comprised $2.0 \mathrm{mSv}$ of effective radiation dose (approximately $35 \mathrm{mGy}$ ) compared with $3.0 \mathrm{mSv}$ of the effective dose in 64-section multidetector row CT angiography ${ }^{5}$ or a conven- 

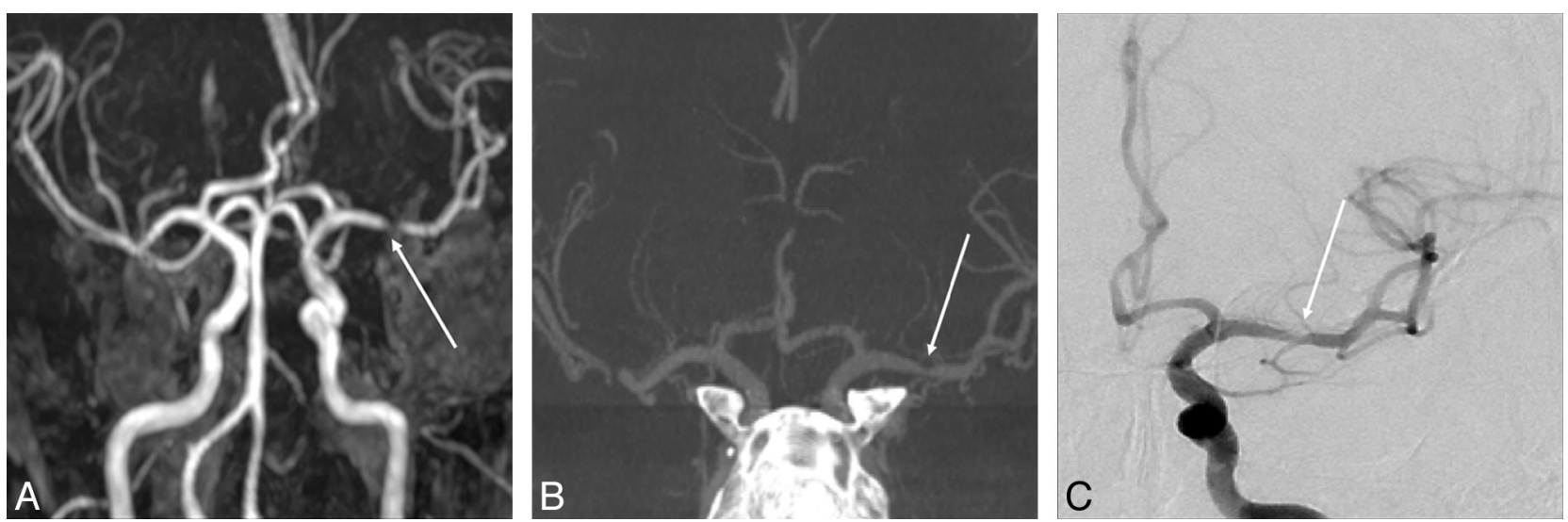

FIG 4. A 55-year-old man was transferred to the emergency department for right-sided hemiparesis. A, Time-of-flight MRA reveals severe stenosis at the left M1 segment (arrow). B, Selective maximum-intensity-projection image of IV FDCT shows $40 \%$ stenosis at the left M1 segment (arrow) with excellent concordance to the DSA reference. C, Anteroposterior DSA of the left internal carotid artery illustrates $40 \%$ stenosis at the left Ml segment (arrow).

tional head CT scan of $60 \mathrm{mGy} .{ }^{24}$ When one assesses the cerebral blood volume for ischemic penumbra area, no additional radiation exposure is necessary. A recent phantom study affirmed the significantly reduced exposure of radiation in IV FDCT through comparative testing with multisection $\mathrm{CT} .{ }^{25}$ With the advance of technology in reducing a higher noise level in a short acquisition mode, high-quality images can be obtained with a much lower effective radiation dose of $0.4 \mathrm{mSv} .^{26}$

IV FDCT has some drawbacks including more motion artifacts and beam-hardening artifacts due to metal. Beam-hardening artifacts can be reduced by using a metal artifacts-reduction algorithm. ${ }^{27}$ The method for reducing motion artifacts due to metal must be studied further.

Several reports have described good correlation between IV FDCT and DSA or CTA. ${ }^{4,28,29}$ However, no objective data analysis has been performed on the reliability of IV FDCT with DSA as a reference. Furthermore, previous studies compared stenotic lesions using different radiologic methods within 30 days. In our investigation, statistical analysis was used for a comparison of IV FDCT and DSA in assessing the stenotic ratio and length. Moreover, the 2 tests were performed simultaneously. Therefore, there is no chance for aggravation of stenosis during the interval time.

There are some shortcomings in this study. First, it was not a randomized controlled study. Second, direct comparison with IV FDCT and other noninvasive methods, including MRA or CTA, was not performed. Third, no statistical analyses were performed on circumferential calcification or distal stenosis between CTA and IV FDCT.

\section{CONCLUSIONS}

IV FDCT can be a reliable alternative noninvasive test for evaluating atherosclerotic stenosis of the major intracranial arteries. Further investigation on the accuracy of IV FDCT with direct comparison with MRA or CTA is needed.

\section{REFERENCES}

1. Wong KS, Li H. Long-term mortality and recurrent stroke risk among Chinese stroke patients with predominant intracranial atherosclerosis. Stroke 2003;34:2361-66

2. Hass WK, Fields WS, North RR, et al. Joint study of extracranial arterial occlusion. II. Arteriography, techniques, sites, and complications. JAMA 1968;203:961-68

3. Dion JE, Gates PC, Fox AJ, et al. Clinical events following neuroangiography: a prospective study. Stroke 1987;18:997-1004

4. Psychogios MN, Buhk JH, Schramm P, et al. Feasibility of angiographic $\mathrm{CT}$ in peri-interventional diagnostic imaging: a comparative study with multidetector CT. AJNR Am J Neuroradiol 2010;31: 1226-31

5. Struffert T, Deuerling-Zheng Y, Kloska S, et al. Flat detector CT in the evaluation of brain parenchyma, intracranial vasculature, and cerebral blood volume: a pilot study in patients with acute symptoms of cerebral ischemia. AJNR Am J Neuroradiol 2010;31:1462-69

6. Samuels OB, Joseph GJ, Lynn MJ, et al. A standardized method for measuring intracranial arterial stenosis. AJNR Am J Neuroradiol 2000;21:643-46

7. Nguyen-Huynh MN, Wintermark M, English J, et al. How accurate is CT angiography in evaluating intracranial atherosclerotic disease? Stroke 2008;39:1184-88

8. Bash S, Villablanca JP, Jahan R, et al. Intracranial vascular stenosis and occlusive disease: evaluation with $\mathrm{CT}$ angiography, MR angiography, and digital subtraction angiography. AJNR Am J Neuroradiol 2005;26:1012-21

9. Layton KF, Huston J 3rd, Cloft HJ, et al. Specificity of MR angiography as a confirmatory test for carotid artery stenosis: is it valid? $A J R$ Am J Roentgenol 2007;188:1114-16

10. Korogi $\mathrm{Y}$, Takahashi $\mathrm{M}$, Mabuchi N, et al. Intracranial vascular stenosis and occlusion: diagnostic accuracy of three-dimensional, Fourier transform, time-of-flight MR angiography. Radiology 1994; 193:187-93

11. Korogi $\mathrm{Y}$, Takahashi $\mathrm{M}$, Nakagawa $\mathrm{T}$, et al. Intracranial vascular stenosis and occlusion: MR angiographic findings. AJNR Am J Neuroradiol 1997; 18:135-43

12. Hirai T, Korogi $Y$, Ono K, et al. Prospective evaluation of suspected stenoocclusive disease of the intracranial artery: combined MR angiography and CT angiography compared with digital subtraction angiography. AJNR Am J Neuroradiol 2002;23:93-101

13. Feldmann E, Wilterdink JL, Kosinski A, et al. The Stroke Outcomes and Neuroimaging of Intracranial Atherosclerosis (SONIA) trial. Neurology 2007;68:2099-106

14. Biondi A, Ricciardi GK, Puybasset L, et al. Intra-arterial nimodipine for the treatment of symptomatic cerebral vasospasm after aneurysmal subarachnoid hemorrhage: preliminary results. AJNR Am J Neuroradiol 2004;25:1067-76

15. Hacke W, Kaste M, Bluhmki E, et al. Thrombolysis with alteplase 3 to 4.5 hours after acute ischemic stroke. $N$ Engl J Med 2008;359: 1317-29

16. del Zoppo GJ, Higashida RT, Furlan AJ, et al. PROACT: a phase II 
randomized trial of recombinant pro-urokinase by direct arterial delivery in acute middle cerebral artery stroke: PROACT Investigators-Prolyse in Acute Cerebral Thromboembolism. Stroke 1998; 29:4-11

17. Jansen $O$, Schellinger $P$, Fiebach J, et al. Early recanalisation in acute ischaemic stroke saves tissue at risk defined by MRI. Lancet 1999; 353:2036-37

18. Riedel $\mathrm{CH}$, Jensen $\mathrm{U}$, Rohr A, et al. Assessment of thrombus in acute middle cerebral artery occlusion using thin-slice nonenhanced computed tomography reconstructions. Stroke 2010;41: $1659-64$

19. Blanc R, Pistocchi S, Babic D, et al. Intravenous flat-detector CT angiography in acute ischemic stroke management. Neuroradiology 2012;54:383-91

20. Kamran M, Nagaraja S, Byrne JV. C-arm flat detector computed tomography: the technique and its applications in interventional neuro-radiology. Neuroradiology 2010;52:319-27

21. Jou $\mathrm{LD}$, Mohamed $\mathrm{A}$, Lee $\mathrm{DH}$, et al. 3D rotational digital subtraction angiography may underestimate intracranial aneurysms: findings from two basilar aneurysms. AJNR Am J Neuroradiol 2007;28:1690-92

22. Trossbach M, Hartmann M, Braun C, et al. Small vessel stents for intracranial angioplasty: in vitro evaluation of in-stent stenoses using CT angiography. Neuroradiology 2004;46:459-63
23. Buhk JH, Lingor P, Knauth M. Angiographic CT with intravenous administration of contrast medium is a noninvasive option for follow-up after intracranial stenting. Neuroradiology 2008;50: $349-54$

24. Shrimpton PC, Hillier MC, Lewis MA, et al. National survey of doses from CT in the UK: 2003. Br J Radiol 2006;79:968-80

25. Bai M, Liu B, Mu H, et al. The comparison of radiation dose between C-arm flat-detector CT (DynaCT) and multi-slice CT (MSCT): a phantom study. Eur J Radiol 2011 Sep 30. [Epub ahead of print]

26. White PM, Gilmour JN, Weir NW, et al. AngioCT in the management of neurointerventional patients: a prospective, consecutive series with associated dosimetry and resolution data. Neuroradiology 2008;50:321-30

27. Prell D, Kyriakou Y, Struffert T, et al. Metal artifact reduction for clipping and coiling in interventional C-arm CT. AJNR Am J Neuroradiol 2010;31:634-39

28. Psychogios MN, Schramm P, Buhk JH, et al. Angiographic CT after intravenous contrast agent application: a noninvasive follow-up tool after intracranial angioplasty and stenting. AJNR Am J Neuroradiol 2010;31:1886-91

29. Struffert T, Kloska S, Engelhorn T, et al. Optimized intravenous flat detector CT for non-invasive visualization of intracranial stents: first results. Eur Radiol 2011;21:411-18 